I. Antépara ${ }^{1}$, A. Roger ${ }^{2}$, N. Bernedo ${ }^{3}$, R. Lleonart ${ }^{4}$, F. Rodríguez ${ }^{5}$, B. Madariaga ${ }^{6}$, J. A. Asturias ${ }^{6}$, L. Begoña ${ }^{6}$, A. Martínez ${ }^{6}$, A. Landeta ${ }^{6}$, M. C. Gómez-Fernández

\title{
Clinical trial to assess tolerability and subrogate efficacy effects of an abbreviated schedule with house dust mites mixture subcutaneous immunotherapy
}

\author{
${ }^{1}$ Department of Allergology, Hospital Universitario de Basurto, Bilbao, Spain \\ ${ }^{2}$ Section of Allergology, Hospital Universitario Germans Trias Pujol, Badalona, Spain \\ ${ }^{3}$ Department of Allergology, Hospital Universitario Araba, Vitoria, Spain \\ ${ }^{4}$ Department of Allergology, Hospital Universitari de Bellvitge, L’Hospitalet de Llobregat, Spain \\ ${ }^{5}$ Department of Allergology, Hospital Universitario Marqués de Valdecilla, Santander, Spain \\ ${ }^{6}$ Department of R\&D, ROXALL Medicina España S.A., Zamudio, Spain
}

\section{KEY WORDS}

House dust mites; DPT/DF mixture;

subcutaneous immunotherapy;

rhinoconjunctivitis; abbreviated schedule.

\section{Corresponding author}

María Cruz Gómez Fernández

Department of R\&D

ROXALL Medicina España S.A.

Parque Científico y Tecnológico de Bizkaia

Edificio 401

48170 Zamudio, Bizkaia, Spain

E-mail: maricruz.gomez@roxall.es

Doi

10.23822/EurAnnACI.1764-1489.140

\begin{abstract}
Summary
Objective. To evaluate the tolerability and efficacy of Dermatophagoides pteronyssinus/Dermatophagoides farinae mixture subcutaneous immunotherapy (SCIT). Methods. Patients received an abbreviated build-up schedule. The aims were: number, percentage, and severity of adverse reactions. Secondary outcomes included: changes in immunoglobulin titers and changes in dose-response skin prick tests. Results. Out of 289 administrations, $17 \%$ elicited any clinically relevant adverse reaction. Most of them were local reactions (LR) (9.4\%) and the rest (7.6\%) were systemic. Significant increases in $\operatorname{sg} G$ and $\operatorname{sig} G 4$ were detected in serum samples. Cutaneous reactivity decreased significantly. Conclusions. SCIT with house dust mites mixture of ROXALL Medicina España S.A. seems to have an acceptable tolerability profile, induces blocking IgG and decreases skin reactivity.
\end{abstract}

\section{Abbreviations}

AEs: Adverse events

AIT: Allergen immunotherapy

AR: Allergic rhinitis

ARIA: Allergic Rhinitis and its Impact on Asthma

ARs: Adverse reactions

DBU: Diagnostic biological unit

DF: Dermatophagoides farinae

PPT: Dermatophagoides pteronyssinus

EAACI: European Academy of Allergy and Clinical Immunology

ELISA: Enzyme-Linked Immune Sorbent Assay

EMA: European Medicines Agency
HDM: House dust mites

ITT: Intention to treat

LR: Local reaction

MedDRA: Medical Dictionary for Regulatory Activities

PP: Per Protocol

SAS: Statistical Analysis Software

SMs: Storage Mites

SPT: Skin prick test

SP: Safety population

SR: Systemic reaction

TSU: Treatment Standardized Unit 


\section{Introduction}

Respiratory allergy brings together a set of conditions with a highly health burden in the world $(1,2)$. Mites cause allergy disease in more than $10 \%$ of globe population and $90 \%$ of the people diagnosed from allergic asthma presents sensitization to domestic mites (3). House dust mites (HDM) are the most abundant aeroallergen in indoor environments, especially in warm and moist areas like Iberian countries (4).

Usually, mites are classified in two vast groups: HDMs belonging to the Pyroglyphidae family and storage mites (SMs) belonging to Glycyphagidae and Acaridae families (5). Dermatophagoides pteronyssinus (DPT) and Dermatophagoides farinae (DF) for HDM, followed by Lepidoglyphus destructor for SMs in a specific area of the north-west of Spain, were the most common species found in an epidemiologic study describing the prevalence of mites' sensitization in four different areas of the country (6). This geographical mites' distribution can imply different sensitization profile in patients of diverse areas and different allergen immunotherapy composition needs $(3,7)$.

It is well ascertained that allergy immunotherapy (AIT) is the unique available therapeutic option to target the disease and not only symptoms (8-11). Thanks to their disease-modifying effect, specific AIT gets immune system tolerance to clinically relevant allergens through triggering specific blocking antibodies, activating mediators and achieving the decrease of the inflammatory response in tissues. Probably, the AIT prescription is considerably lower than $10 \%$ of patients with AR or asthma $(12,13)$.

According to EMA guidelines, mixture of different allergenic sources is only recommended when they are taxonomically related (14). In the case of DPT and DF mixture, the similarity and cross-reactivity is so high, between $80-90 \%(15,16)$ that it could be enough to receive unique vaccine with one of these two allergen extracts. As a consequence of the enormous homology, any of the two allergenic sources could be proposed as the representative homologous specie (17).

However, a considerable number of clinicians remains prescribing HDM mixture (50\% DPT and 50\% DF) mainly based on patient's sensitization results $(18,19)$.

Therefore, an open multicentre clinical trial in adult patients with allergic rhinitis (AR), (with or without asthma) using a standardized native depot HDM mixture extract for subcutaneous immunotherapy was conducted. The principal objective was to establish the tolerability and safety of an abbreviated treatment schedule in patients sensitized to HDM.

\section{Materials and methods}

\section{Study design and ethical considerations}

Five hospitals in Spain collaborated in this open, multicentre and phase I clinical trial. Likewise, the study was conducted in accor- dance with the principles of the Declaration of Helsinki and the ICH guideline on Good Clinical Practice. It was approved by relevant ethics committees and by the Spanish regulatory authorities, (EudraCT 2015-004712-38). Prior to their participation, written informed consent was given by every patient.

\section{Study population}

Patients had to meet the following criteria: age 18-60 years, clinical history of perennial AR due to HDM for at least 2 years prior to the study inclusion, a positive skin prick test to DPT or DF (wheal diameter $\geq 3 \mathrm{~mm}$ ) and specific immunoglobulin $\mathrm{E}$ (sIgE) against DPT or DF levels $\geq 0.7 \mathrm{kUa} / \mathrm{L}$ determined by ImmunoCAP ${ }^{\circledR}$ (Thermo Fisher Scientific, Uppsala, Sweden). Results of SPT performed within 12 months prior to the inclusion were accepted. Only patients with concurrent mild asthma were allowed to participate.

The following were defined as exclusion criteria: to have received immunotherapy against HDM or a cross-reactive allergen in the 5 years prior the study inclusion, current administration of immunotherapy for any other allergen, moderate to severe asthma, forced expiratory volume in $1^{\text {st }}$ second $(\mathrm{FEV} 1)<70 \%$, clinically relevant perennial sensitization different of HDM. The following conditions were additional exclusion criteria: history of anaphylaxis, chronic urticaria, moderate to severe atopic dermatitis; immunological, cardiac, renal or hepatic diseases; current treatment with immunosuppressants, anti-IgE, tricyclic antidepressants, psychotropic drugs, beta-blockers, or angiotensin-converting enzyme inhibitors and pregnant or breast-feeding women.

\section{Study interventions}

Patients were treated with a native depot mixture of DPT and DF subcutaneous treatment, (Allergovac ${ }^{\circledR}$ Depot, ROXALL Medicina España S.A., Zamudio, Spain) consisting of two different strengths. The abbreviated build-up schedule comprised 6 doses at weekly intervals ( \pm 2 days): 3 doses $(0.2,0.5$ and $1 \mathrm{~mL})$ from vial 2 (100 Treatment Standardized Units (TSU)/mL), and 3 subsequent administrations $(0.2,0.5$ and $1 \mathrm{~mL})$ from vial 3 . The last dose of the increasing period, $1 \mathrm{~mL}$ of vial 3, $1000 \mathrm{TSU} / \mathrm{mL}$, was the target maintenance dose and was administered at monthly intervals, during one trimester, being the whole treatment duration of 17 weeks. The concentration of the major HDM allergens for group 1 were: Der p1 $0.44 \mu \mathrm{g} / \mathrm{mL}$ and $0.34 \mu \mathrm{g} / \mathrm{mL}$ Der f1 and for group 2 were Der p2 $0.69 \mu \mathrm{g} / \mathrm{mL}$ and $0.45 \mu \mathrm{g} /$ $\mathrm{mL}$ Der $\mathrm{f} 2$. Some dose schedule variations were allowed in the event of adverse reactions according to the standards for practical allergen-specific immunotherapy recommendations (20).

\section{Outcome measures}

In this study all adverse events (AEs) were registered for tolerability assessment. The primary outcome was the incidence of adverse reactions (ARs), recorded at participating sites during the 
30 minutes after each vaccine administration. In addition, ARs were also collected by reviewing the patients' diaries designed to register any unpleasant experience outside immunotherapy units and by telephone calls. ARs were defined as all noxious and unintended responses to any dose of the investigational allergen vaccine administered. These reactions were classified as immediate (within 30 minutes after the vaccine administration) or delayed (> 30 minutes after vaccine administration).

In the same way, adverse reactions were classified as local (LR, reactions taking place at the arm where vaccine was administered), or systemic reactions (SRs, generalised symptoms taking place far away from the administration site). According to LRs extension, we consider clinically significant the immediate $L R \geq 5 \mathrm{~cm}$ and the delayed $L R \geq 10 \mathrm{~cm}$ or those implying a dose modification in the next administration. Additionally, LRs were described as diffuse inflammation, redness, erythema, local painfulness, pruritus or reaction in injection site (when two or more local symptoms took place simultaneously). SRs were classified by the investigators according to the European Academy of Allergy and Clinical Immunology EAACI guidelines (20) and also by the Medical Dictionary for Regulatory Activities (MedDRA).

Dose-response skin prick test (SPT) was performed using four increasing concentrations of HDM mixture extract (100, 1.000, 10.000 and $100.000 \mathrm{DBU} / \mathrm{mL}$, Diagnostic Biologic Units) as well as positive (histamine $10 \mathrm{mg} / \mathrm{mL}$ ) and negative (saline) controls. Titrated skin prick test for basal and final visits, were provided by ROXALL Medicina España S.A. to the study participants. The batch used for the whole study population was the same. The change in cutaneous reactivity (wheal area in $\mathrm{mm}^{2}$ ) from baseline to the final visit was measured.

Regarding the immunological effects' assessment, serum samples were obtained at baseline and final visits to determinate immunoglobulin levels (sIgE, sIgG and sIgG4) against DPT and DF whole extract by ELISA (Enzyme-Linked Immune Sorbent Assay) as previously described in Sola J. et al. (2015) (21). Samples were frozen and sent to ROXALL's central laboratory for bioanalysis in accordance with Good Laboratory Practices.

Moreover, specific immunoglobulin titters IgE against Der p1, Der $\mathrm{p} 2$ and Der $\mathrm{p} 10$ were analysed at baseline and final visit by ImmunoCAP ${ }^{\circledR}$ (Thermo Scientific, Uppsala, Sweden).

\section{Statistical methods}

We described three populations: safety population (SP), patients who received at least one dose of treatment, intention-to-treat (ITT) population, patients who met all inclusion/exclusion criteria, received at least one dose of treatment, and had available data on subrogate efficacy variables, and the per-protocol (PP) population, patients who met previous criterial and moreover achieved their target maintenance dose and completed the study without any major protocol deviation.
Tolerability and safety were analysed using descriptive statistics. Categorical variables were described by absolute and relative frequencies and in continuous variables the mean and the standard deviation were applied.

Changes in immunoglobulin levels and SPT values from baseline to final visit, were analysed by means of the Wilcoxon non-parametric test for paired samples. A bilateral statistical significance level of 0.05 was displayed to all statistical tests. Statistical analyses were performed using the Statistical Analysis software (SAS) version 9.4. Sample size was calculated considering a percentage of ARs of $22,9 \%$ (22). Establishing a confidence interval of $95 \%$ with a precision of \pm 4 percentage unit and assuming a 5\% of drop outs, the number of patients to provide adequate data on the primary endpoint was 42 .

\section{Results}

\section{Descriptive data}

A total of 44 patients were recruited from August 2016 to April 2017. Out of them, one was a screening failure and other one removed the consent prior to start treatment so, 42 patients were assigned to receive HDM mixture AIT and were analysed in SP. Additionally, in this study there were 6 early discontinuations: 1 due to $\mathrm{AE}$, defined as hearing loss, 1 for surgery intervention, 3 for loss of follow up and 1 for change in the residence address. Rhinoconjunctivitis secondary to sensitization to Dermatophagoides was confirmed in each participant by allergy diagnostic tests and a rigorous clinical history. Regarding other sensitizations, the percentage of patients sensitized to grass pollen was $7.14 \%$, to weed pollen $4.76 \%$, and to tree pollen $2.38 \%$. However, these aeroallergen sensitizations were not clinically relevant or did not interfere with the collecting data period. ITT population included 37 patients since 5 were excluded due to the absence of data on immunoglobulins or dose-response SPT at final visit. Finally, 31 patients remained in the PP population, mainly as a result of major protocol deviations. Patient's distribution is shown in figure 1. Most patients showed sIgE class $\geq 4$ against whole DPT and DF extract: $38.1 \%$ and 50\% respectively. Subjects' baseline clinical characteristics and sIgE profile is presented in table $\mathbf{I}$.

\section{Tolerability and safety}

All patients presented at least $1 \mathrm{AE}$ in the study, being classified the majority of them as mild to moderate intensity. The most frequent reported AEs were, injection site reaction, headache, upper respiratory tract infections and digestive system disorders. All $\mathrm{AE}$ were non-serious and the vast majority were resolved with symptomatic medication.

ARs were summarized in table II and III. Out of 289 dose administrations, $6(2.1 \%)$, were considered as clinically relevant immediate LR, and 21 (7.3\%) clinically relevant delayed LRs. All of them described as injection site reaction. 
Figure 1 - Study flow chart.

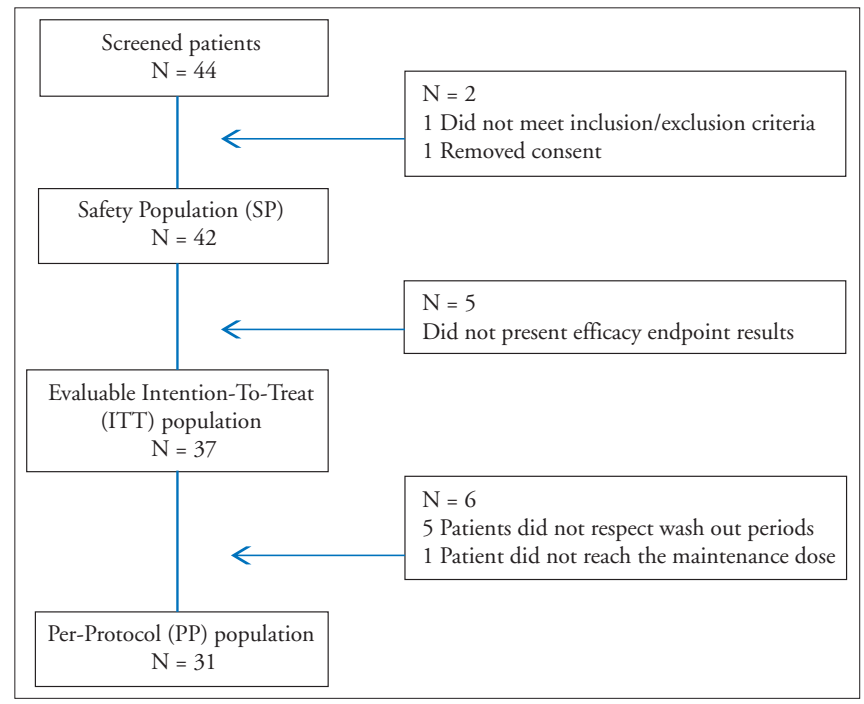

Regarding systemic reactions, 22 SRs (7.6\% of dose administrations) were recorded; six grade $0(2.1 \%)$, fifteen $(5.2 \%)$ grade I and one grade II $(0.3 \%)$. There were no systemic reactions grades III or IV. All of them are described in table III.

SRs were resolved with symptomatic treatment or a change in the next administration dose. All patients recovered of the ARs at the end of the study. One patient in spite of the dose modifications performed in the schedule, did not reach the maintenance dose established in the study protocol due to adverse reactions. This patient with the worst systemic reaction, described as delayed rhinitis with dyspnoea responded to the symptomatic treatment with beta 2 blockers, inhaled corticosteroids and antihistamines. At basal period, she/he presented class 4 levels of sIgE against DPT and DF. However, papule areas before immunotherapy treatment showed a size similar to the mean of the sample population.

Clinically relevant changes in blood count and biochemistry parameters were not observed in any patient after receiving immunotherapy treatment.

\section{Immunoglobulin levels}

Statistically significant increases in serum sIgG and sIgG4 titters against DPT and DF whole extract at final visit were observed compared with basal visit (both $\mathrm{p}<0.0001$; Wilcoxon test). Serum sIgE levels to DPT and DF slightly decreased at final visit, achieving statistical significance $(\mathrm{p}<0.0002$; Wilcoxon test) (figure 2). On contrary, a statistically significant increases in serum sIgE against Der p1 and Der p2 at final visit were observed in comparison with basal visit $(\mathrm{p}<0.0001$ and $\mathrm{p}<0.02$, respectively; Wilcoxon test). As it was expected, these results were maintained in PP population.
Table I - Patients' baseline clinical characteristics.

\begin{tabular}{|c|c|}
\hline Baseline characteristics & \\
\hline Number of patients $(S P)$ & 42 \\
\hline Age (years), mean $\pm(S D)$ & $33.6 \pm 9.1$ \\
\hline Women $n(\%)$ & $21(50.0)$ \\
\hline $\begin{array}{l}\text { Race } n(\%) \\
\text { Caucasians } \\
\text { Hispanics } \\
\text { Arabs } \\
\text { Asians }\end{array}$ & $\begin{array}{l}31(73.8) \\
7(16.7) \\
2(4.8) \\
2(4.8)\end{array}$ \\
\hline $\begin{array}{l}\text { Rhinitis ARIA classification (32) } \\
\text { Intermittent mild n (\%) } \\
\text { Persistent mild n (\%) } \\
\text { Intermittent moderate-severe } n(\%) \\
\text { Persistent moderate-severe } n(\%)\end{array}$ & $\begin{array}{l}1(2.4) \\
9(21.4) \\
2(4.8) \\
30(71.4)\end{array}$ \\
\hline $\begin{array}{l}\text { Main concomitant allergic condition } \\
\text { Asthma n (\%) }\end{array}$ & $7(16.7)$ \\
\hline Time from diagnostic (years), mean $\pm(S D)$ & $4.7 \pm(8.4)$ \\
\hline$(\mathbf{B M I}), \mathbf{K g} / \mathbf{m}^{2}$ mean $\pm(S D)$ & $24.7(4.85)$ \\
\hline $\begin{array}{l}\text { Vital signs mean } \pm(S D) \\
\text { Systolic blood pressure, } m m H g \\
\text { Diastolic blood pressure, } m m H g \\
\text { Heart rate, bpm }\end{array}$ & $\begin{array}{c}114.7(14.1) \\
71.7(10.6) \\
71.6(9.2)\end{array}$ \\
\hline $\begin{array}{c}\text { sIgE DPT CAP class } n(\%) \\
2 \\
3 \\
4 \\
5\end{array}$ & $\begin{array}{l}1(2.4) \\
9(21.4) \\
16(38.1) \\
16(38.1) \\
\end{array}$ \\
\hline $\begin{array}{c}\text { sIgE DF CAP class } n(\%) \\
2 \\
3 \\
4 \\
5\end{array}$ & $\begin{array}{l}1(2.4) \\
11(26.2) \\
21(50.0) \\
9(21.4)\end{array}$ \\
\hline
\end{tabular}

\section{Cutaneous reactivity}

Mean values of wheal area in $\mathrm{mm}^{2}$ were significantly reduced at final visit compared with baseline in each one of the four tested vials against HDM mixture (figure 3). Moreover, a statistical significance was achieved with all tested vials ( $\mathrm{p}<0.04$; Wilcoxon test from vial 1 to vial 4). These cutaneous results were also reproduced in the PP population.

\section{Discussion}

Despite the fact of great homology between DPT and the rest of the mites belonging to the family Pyroglyphidae, $(15,16,23)$ 
Table II - Summary of adverse reactions in SP.

\begin{tabular}{|c|c|c|c|}
\hline & Schedule Phase & $\begin{array}{l}\text { Patients number n (\%) } \\
42(100 \%)\end{array}$ & $\begin{array}{l}\text { Administered doses n (\%) } \\
289(100 \%)\end{array}$ \\
\hline Clinically relevant immediate $L R s$ & Initiation Phase & $5(11.9 \%)$ & $6(2.1 \%)$ \\
\hline Clinically relevant delayed LRs & Initiation Phase & $12(28.6 \%)$ & $21(7.3 \%)$ \\
\hline Grade 0 & Initiation Phase & $6(14.3 \%)$ & $6(2.1 \%)$ \\
\hline Grade I & Initiation Phase & $9(21.4 \%)$ & $15(5.2 \%)$ \\
\hline Grade II & Initiation Phase & $1(2.4 \%)$ & $1(0.3 \%)$ \\
\hline
\end{tabular}

$n(\%)$ number and percentage of adverse reactions, LR (local reaction) and SP (safety population).

Table III - Description of systemic adverse reactions by administration doses.

( $\mathrm{N}=289$ doses administered $)$

\begin{tabular}{llll}
\hline & Number & Description \\
\hline Grade 0 & $6(2.1 \%)$ & 2 & Headache \\
& 3 & General desconfort + nausea + dizziness \\
& 1 & Non-specific cough \\
\hline Grade I & $15(5.2 \%)$ & Conjunctivitis \\
& 4 & Dermatitis \\
& 2 & Allergic rhinitis \\
& 1 & Urticaria \\
& 1 & Generalized pruritus \\
& 2 & Pruritus out of the injection site \\
& 1 & Erythema out of the injection site \\
& 1 & Throat irritation \\
Grade II & 1 & Pharyngitis \\
& $1(0.3 \%)$ & Allergic cough \\
\hline
\end{tabular}

$n$ (\%) number and percentage of adverse reactions. (ARs) Adverse Reactions.

allergy clinicians commonly prescribe mixed vaccines to treat patients polysensitized to mites. As it was mentioned, the most common mites with positive results in diagnostic prick tests performed in patients with respiratory allergy in Spain, were DPT, DF and Lepidoglyphus destructor (6), excluding the islands. As a consequence, immunotherapy containing a mixture of DPT and DF is frequently prescribed, although a recent publication did not find differences in efficacy between two commercial mites' extracts: one with DPT as single source and other with a mixture of DPT and DF (50/50) (24).
ROXALL Medicina España S.A. (formerly Bial) conducted two clinical trials with Allergovac ${ }^{\circledR}$ Depot native DPT 100\%. A placebo-controlled Phase I study to evaluate three different buildup schedules (22) and a dose finding randomized controlled trial to compare the efficacy of five different doses (25). In both studies, the tolerability profile of the abbreviated schedule could be ascertained as good. However, this evidenced data must be interpreted with caution when they are extrapolated to another marketed product with HDM mixture. A new safety and tolerability trial, with the same schedule and a treatment containing 
Figure 2 - Changes in specific immunoglobulins against HDM.

Changes in specific immunoglobulins against HDM

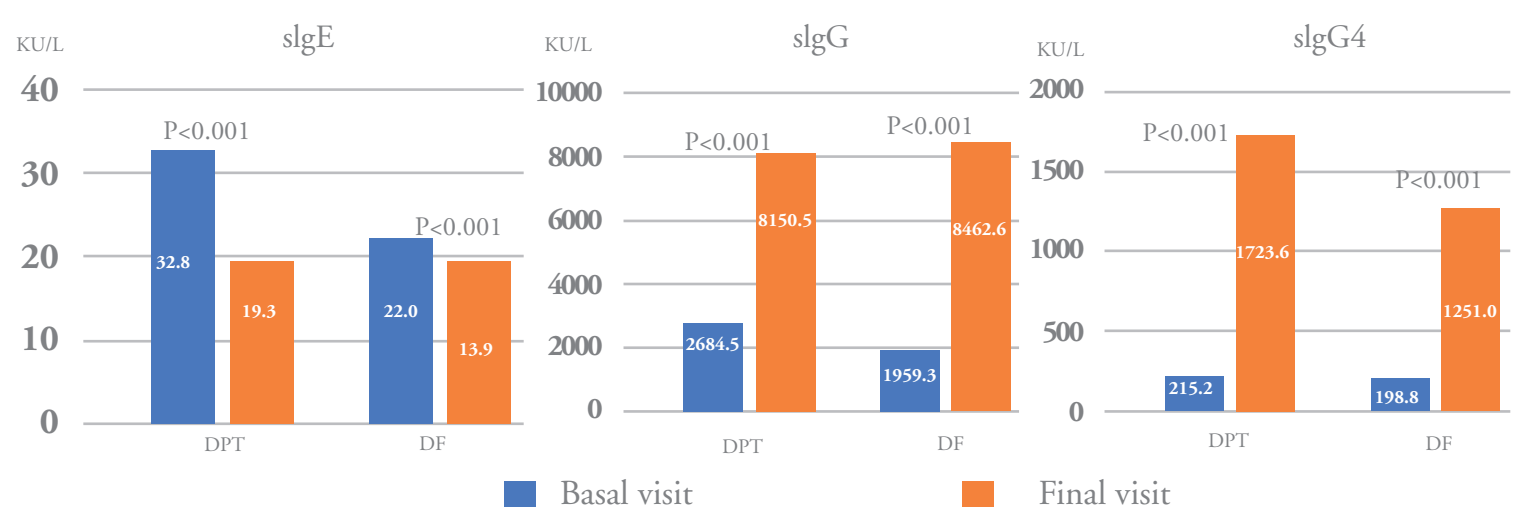

Changes in specific immunoglobulins against HDM. Corresponding p values according to Wilcoxon test are indicated.

Figure 3 - Change in mean wheal area after SPT with HDM mixture.

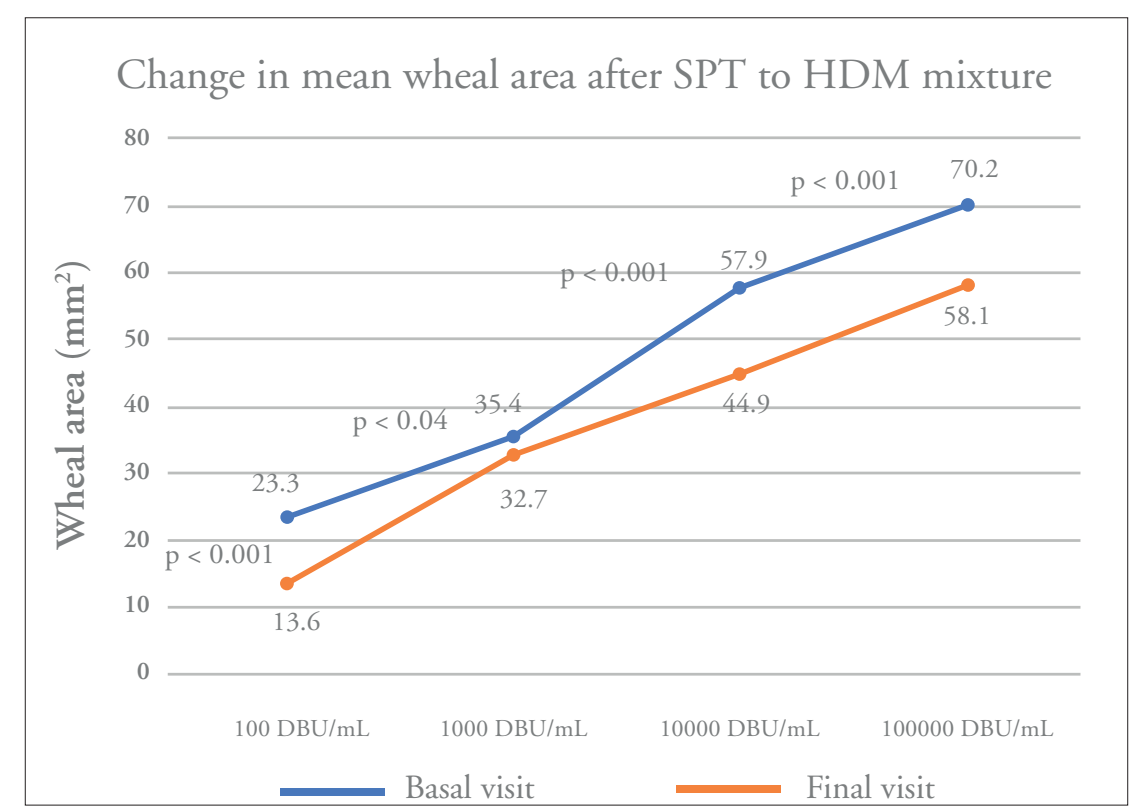

Change in mean wheal area after SPT with HDM mixture at final visit versus baseline. p values according to Wilcoxon test are indicated.

a DPT/DF mixture (50/50), was designed in order to avoid this extrapolation.

The percentage of systemic ARs with the vaccine under study was slightly higher than with the DPT $100 \%$ vaccine used in previous ROXALL clinical trials. In current study, a $7.6 \%$ of systemic ARs was described versus $4.8 \%$ in the phase I study
(22) and 3.8\% with the group containing the commercial dose, in the dose finding trial (25). In comparison with other similar marketed products, the encountered results are in a similar range, thus a study conducted by Tabar et al. (26) showed a systemic ARs percentage of $8.8 \%$ by patient. In another clinical trial with a DPT $100 \%$ formulation, a $9.8 \%$ of ARs was report- 
ed (27). In a comparative study of two schedules with HDM depot native immunotherapy (28), the percentage of ARs with conventional schedule reached $13.8 \%$ and $10.7 \%$ with cluster scheme. Additionally, in similar designed studies, but with different extracts composition apart from HDM, the percentage of ARs was even higher reaching in some cases 21\% (29).

This depot formulation induced an early immunological response, confirmed by statistically significant increments of sIgG and sIgG4 levels against DPT and DF, after approximately 3 months of therapy. Similar results could be observed in other studies, where a fast increase in $\operatorname{sgG}$ and $\operatorname{sgG} 4$ can be associated with the effect of blocking IgE-binding to allergens and immune response modification $(26,30,31)$. These results are in line with the immunologic and skin prick test outcomes observed in previous studies with DPT 100\% (Allergovac ${ }^{\circledR}$ Depot, ROXALL Medicina España S.A., Zamudio, Spain) (22, 25). Regarding sIgE determination against Der $\mathrm{p} 1$ and Der $\mathrm{p} 2$, surprisingly a statistically significant increased at final visit was observed. These increases could be attributed to the effect of other allergens different to Der $\mathrm{p} 1$ and Der $\mathrm{p}$, with relevance in the study patients' immune response.

Considering the cutaneous reactivity to the causal allergens, a statistically significant reduction in immediate skin response to the different concentrations of DPT and DF combination was observed, showing a decrease in the mean papule size produced by each concentration tested. This result is in the line of another clinical trial with an extract of HDM mixture after a short administration of specific immunotherapy (26).

\section{Conclusions}

Given the heterogeneity in participants, allergens, schedules, dosing treatment and adverse reactions reporting methodology, it is difficult to compare tolerability results between different available studies. However, this clinical trial shows that the assayed abbreviated schedule with native depot HDM mixture, (Allergovac ${ }^{\circledR}$ Depot ROXALL Medicina España), has an acceptable tolerability profile. Moreover, preliminary positive efficacy response can be observed due to a significant immunological and cutaneous reactivity changes in subjects suffering from allergic rhinoconjunctivitis. These promising results should be worth to be confirmed in a larger controlled clinical trial.

\section{Acknowledgements}

The authors would like to thank participating hospitals and the inestimable help of all collaborator investigators like: Ignacio Jáuregui, Nathalie Depreux, Clara Padró, Blanca Andrés, Marta Velasco, Marta Frías, Verónica Salinas, Ma Cruz Santín, Ma Jesus Ortiz and Eva González-Morchón.

\section{Fundings}

This study was supported by ROXALL Medicina España S.A.

\section{Conflict of interests}

Ignacio Antépara has received research fees from, Novartis, GSK, Astra-Zeneca, Sanofiand Bial. Albert Roger has received research fees from Roxall, Allergy Therapeutics, Stallergenes, Leti, Hal, Merck, Diater. Consultant fees: Allergy Therapeutics, Stallergenes, Merck. Speaker fees: Roxall, Allergy Therapeutics, Leti, Merck. Nagore Bernedo has received consultant fees from ALK and Allergy Therapeutics, speaker fees from Roxall and research fees from Merck. Fernando Rodríguez has received research fees from GSK, ALK and Roxall. Speaker fees: GSK, Novartis, Astra-Zeneca and Chiesi. Begoña Madariaga, Juan A Asturias, Leire Begoña, Alberto Martínez, Araitz Landeta and María C Gómez are fulltime employees of ROXALL Medicina España S.A. Ramón Lleonart has no conflicts of interest to declare. The authors have no other relevant affiliations or financial involvement with any organization or entity with a financial interest in or financial conflict with the subject matter or materials discussed in the manuscript apart from those disclosed.

\section{References}

1. Fassio F, Guagnini F. House dust mite-related respiratory allergies and probiotics: A narrative review. Clin Mol Allergy 2018;16(1):1-7.

2. Tortajada-Girbés M, Mesa del Castillo M, Larramona H, et al. Evidence in immunotherapy for paediatric respiratory allergy: Advances and recommendations. Allergol Immunopathol (Madr) 2016;44(Suppl 1):1-32.

3. Li L, Qian J, Zhou Y, et al. Domestic mite-induced allergy: Causes, diagnosis, and future prospects. Int J Immunopathol Pharmacol 2018;32:1-8.

4. Pereira C, Valero A, Loureiro C, et al. Iberian study of aeroallergens sensitisation in allergic rhinitis. Eur Ann Allergy Clin Immunol 2006,38(6):186-94.

5. Gafvelin G, Johansson E, Lundin A, et al. Cross-reactivity studies of a new group 2 allergen from the dust mite Glycyphagus domesticus, Gly d 2, and group 2 allergens from Dermatophagoides pteronyssinus, Lepidoglyphus destructor, and Tyrophagus putrescentiae with recombinant allergens. J Allergy Clin Immunol 2001;107(3):511-8.

6. Morales $\mathrm{M}$, Iraola $\mathrm{V}$, Leonor JR, et al. Different sensitization to storage mites depending on the co-exposure to house dust mites. Ann Allergy Asthma Immunol 2015;114(1):36-42.

7. Vidal C, Lojo S, Juangorena M, et al. Association between asthma and sensitization to allergens of Dermatophagoides pteronyssinus. J Investig Allergol Clin Immunol 2016;26(5):304-9.

8. Nurmatov U, Dhami S, Arasi S, et al. Allergen immunotherapy for allergic rhinoconjunctivitis: a systematic overview of systematic reviews. Clin Transl Allergy 2017;7(1):24.

9. Shamji MH and Durham SR. Mechanisms of allergen immunotherapy for inhaled allergens and predictive biomarkers. J Allergy Clin Immunol 2017;140(6):1485-98. 
10. Mösges R, Valero Santiago A, Allekotte S, et al. Subcutaneous immunotherapy with depigmented-polymerized allergen extracts: A systematic review and meta-analysis. Clin Transl Allergy 2019;9(1):1-15.

11. Klimek L, Fox GC and Thum-Oltmer S. SCIT with a high-dose house dust mite allergoid is well tolerated : safety data from pooled clinical trials and more than 10 years of daily practice analyzed in different subgroups. Allergo J Int 2018;27(5):131-9.

12. Jutel M, Agache I, Bonini S, et al. International consensus on allergy immunotherapy. J Allergy Clin Immunol 2015;136(3):556-8.

13. Mahler V, Klein C, Sager A, et al. House dust mite-specific immunotherapy with two licensed vaccines: Outcome under clinical routine conditions. Immun Inflamm Dis 2017;5(2):132-40.

14. Roberts G, Pfaar O, Akdis CA, et al. EAACI Guidelines on Allergen Immunotherapy : Allergic rhinoconjunctivitis 2018;73(4):765-98.

15. Thomas WR. House Dust Mite Allergens: New discoveries and relevance to the allergic patient. Curr Allergy Asthma Rep 2016;16(9):69.

16. Yang Y, Zhu R, Huang N, et al. The Dermatophagoides pteronyssinus molecular sensitization profile of allergic rhinitis patients in central China. Am J Rhinol Allergy 2018;32(5):397-403.

17. European Medicines Agency. Committee for Medicinal Products for Human Use (CHMP). Guidelines on allergen products: producttion and quality issues. CHMP/BWP/304831/2007, London, UK, 2008.

18. Vidal C, Enrique E, Gonzalo A, et al. Diagnosis and allergen immunotherapy treatment of polysensitised patients with respiratory allergy in Spain: an Allergists' Consensus. Clin Transl Allergy 2014;4:1-12.

19. Wahn U, Calderón MA and Demoly P. Real-life clinical practice and management of polysensitized patients with respiratory allergies: a large, global survey of clinicians prescribing allergen immunotherapy. Expert Rev Clin Immunol 2017;13(3):283-9.

20. Alvarez-Cuesta E, Bousquet J, Canonica GW, et al. EAACI, Immunotherapy Task Force. Standards for practical allergen-specific immunotherapy. Allergy 2006;61(Suppl 82):1-20.

21. Sola J, Sánchez V, Landeta A, et al. A Phase I clinical trial with subcutaneous immunotherapy vaccine of Timothy grass pollen extract according to EMA guidelines. Immunotherapy 2015;7(4):343-52.

22. Fernández De Rojas D, Ercoreca IA, Tellechea AP, et al. Phase I study of subcutaneous allergen immunotherapy with Dermatophagoides pteronyssinus in patients with allergic rhinoconjunctivitis with or without asthma. Immunotherapy 2015;7(2):89-99.
23. Shafique RH, Klimov PB, Inam M, et al. Group 1 allergen genes in two species of house dust mites, Dermatophagoides farinae and D. pteronyssinus (Acari: Pyroglyphidae): Direct sequencing, characterization and polymorphism. PLoS One 2014;9(12):1-18.

24. Li J, Wu Y, Yang Y, et al. The efficacy and safety of two commercial house dust mite extracts for allergic rhinitis: a head-to-head study. Int Forum Allergy Rhinol 2019;9(8):876-82.

25. Moreno V, Alvariño M, Rodríguez F, et al. Randomized dose response study of subcutaneous immunotherapy with a Dermatophagoides pteronyssinus extract in patients with respiratory allergy. Immunotherapy 2016;8:265-77.

26. Tabar AI, González Delgado P, Sánchez Hernández C, et al. Phase II/III clinical trial to assess the tolerability and immunological effect of a new updosing phase of Dermatophagoides mix-based immunotherapy. J Investig Allergol Clin Immunol 2015;25(1):40-6.

27. Tabar AI, Echechipía S, García BE, et al. Double-blind comparative study of cluster and conventional immunotherapy schedules with Dermatophagoides pteronyssinus. J Allergy Clin Immunol 2005;116(1):109-18.

28. Fan Q, Liu X, Gao J, et al. Comparative analysis of cluster versus conventional immunotherapy in patients with allergic rhinitis. Exp Ther Med 2017;13(2):717-22.

29. Pfaar O, Wolf H, Klimek L, et al. Immunologic effect and tolerability of intra-seasonal subcutaneous immunotherapy with an 8-day up-dosing schedule to 10.000 standardized quality-units: a double-blind randomized, placebo-crontrolled trial. Clin Ther 2012;34(10):2072-81.

30. Jutel M, Rudert M, Kreimendahl F, et al. Efficacy and tolerability of a house dust mite allergoid in allergic bronchial asthma: A randomized dose-ranging trial. Immunotherapy 2018;10(13):1149-61.

31. Roger A, Depreux N, Jurgens Y, et al. A novel microcrystalline tyrosine-adsorbed, mite-allergoid subcutaneous immunotherapy: 1-year follow-up report. Immunotherapy 2016;8(10):1169-1174.

32. Brozek JL, Bousquet J, Baena-Cagnani CE, et al. Global Allergy and Asthma European Network; Grading of Recommendations Assessment, Development and Evaluation Working Group. Allergic Rhinitis and its Impact on Asthma (ARIA) guidelines: 2010 revision. J Allergy Clin Immunol 2010;126(3):466-76. 